Results In the 12 weeks post-diagnosis, for a large majority of respondents there was a reduction in the expected number of casual partners who would be infected: $76 \%$ of participants eliminated risk of onward transmission entirely. However, a small proportion still presented a transmission risk. Overall, reductions in HIV transmission risk behaviour post-diagnosis would have reduced estimated secondary transmission during primary HIV infection (PHI) from been $33(23-37)$ to $12(6-14)$ - a reduction of $62 \%(32 \%-83 \%)$. Diagnosis after PHI produces a more modest reduction in transmission by missing the high-infectivity period.

Conclusions Diagnosis of PHI can produce a large proportionate reduction in HIV-transmission events by reducing transmission-risk behaviour. Due to the high infectivity but short duration of primary infection, even short-term behaviour change can significantly reduce transmission. Later diagnosis is less effective, whilst early diagnosis requires frequent or highly-targeted testing. Whilst further work is required to determine the costs of different testing strategies, our quantification of the number of infections averted is an essential component of an assessment of the cost-effectiveness of strategies to increase early diagnoses of HIV infection.

\section{1-S11.04 TARGETING THE USE OF HIV RNA SCREENING TO MAXIMISE YIELD AND MINIMISE COST: NYC HEALTH DEPARTMENT STD CLINICS, 2008-2010}

\section{doi:10.1136/sextrans-2011-050109.64}

${ }^{1} \mathrm{~S}$ Blank, ${ }^{2} \mathrm{C}$ Borges, ${ }^{2} \mathrm{~A}$ Kowalski, ${ }^{2} \mathrm{~S}$ Sebiyam, ${ }^{2} \mathrm{M}$ Sweeney. ${ }^{1} \mathrm{NYC}$ DOHMH / CDC, New York, USA; ${ }^{2}$ NYC DOHMH, New York, USA

Background Nucleic acid amplification testing (NAAT) is an important tool for identifying acute HIV infection (AHI), a period of high infectivity when antibody is undetectable. NAAT pooling methods (pNAAT) help contain the costs of screening for AHI. In 2008 NYC STD clinics began routine pNAAT screening for all rapid antibody negative specimens; it was standard of care in all nine clinics by 2009. A pattern of risk factors among AHI cases detected during universal screening suggested the feasibility of using targeted screening to maximise the yield of AHI cases detected while minimising costs of screening.

Methods Using medical record data, we reviewed cases of AHI diagnosed in nine NYC STD clinics for 2008-2009. From these we developed targeting criteria for AHI screening, and compared yields and costs before and after targeting.was implemented.

Results Targeted screening began in May 2010 and included the following risk criteria: MSM, females who have had sex with MSM,

\section{NON-SCHOOL DAY}

\section{Sexual intercourse}

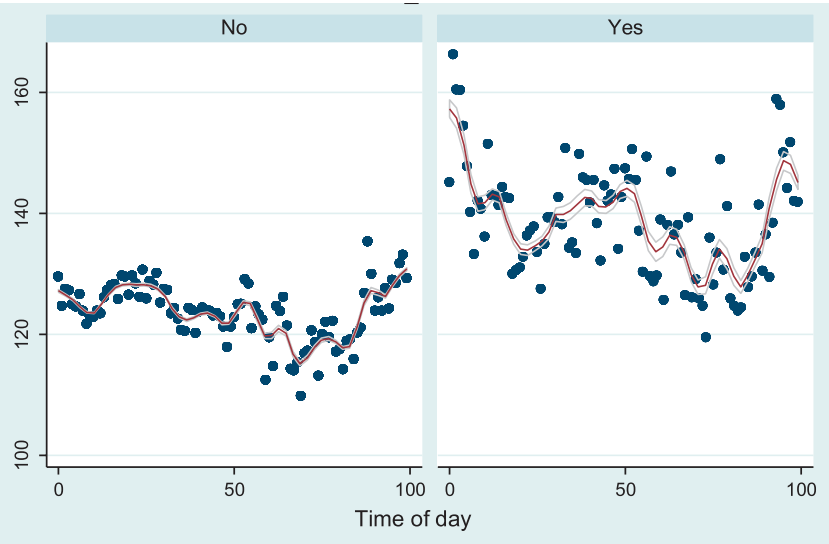

sex with an injection drug user, exchange sex for money or drugs, shared injection drug works, or recent victim of sexual assault. Prior, 42696 specimens were screened by pNAAT from June through December 2009, yielding 23 AHI cases (5.4 cases/10 000 specimens). Of these cases, there were 21 males, including 15 who have sex with men (MSM) (71\%, 15/21), 1 female, and 1 transgender. The mean age for patients was 30 years; racial/ethnic breakdown was: $57 \%$ Black, 39\% Hispanic, 13\% white, 4\% other. Subsequently, 5280 specimens were screened by pNAAT from June through December 2010 , representing an $88 \%$ decrease in testing compared to the same period during the previous year. A total of $18 \mathrm{AHI}$ cases (34.1/10 000 specimens) were detected; all were MSM. The mean age was 29 years and racial/ethnic breakdown was: 44\% Black, 28\% Hispanic, 28\% white, 5\% Asian. Cost data are provided in Abstract O1-S11.04 table 1

Abstract 01-S11.04 Table 1 Cost effectiveness of targeted AHI screening

\begin{tabular}{lll}
\hline & Universal & Targeted \\
\hline Average pooled AHI specimens per month & 5700 & 770 \\
Annual cost of pooled AHI screening & $\$ 650000$ & $\$ 91296$ \\
Annual yield & 33 & 35 \\
Average total cost per month & $\$ 54167$ & $\$ 7608$ \\
Average cost per AHI case identified & $\$ 19697$ & $\$ 2608$ \\
\hline
\end{tabular}

Conclusion AHI screening increases case detection compared to using antibody tests alone. After initial investment in the effort, we were able to cut the cost per case identified by over sevenfold. This approach may make AHI screening more feasible/affordable in settings with patients at very high risk of newly-acquiring HIV.

\section{1-S11.05 ADOLESCENT SEXUAL INTERCOURSE AND NEIGHBOURHOOD SOCIAL DISORDER}

doi:10.1136/sextrans-2011-050109.65

${ }^{1} \mathrm{~S}$ Wiehe, ${ }^{2} \mathrm{M} \mathrm{P}$ Kwan, ${ }^{1} \mathrm{~S}$ Hoch, ${ }^{1} \mathrm{~B}$ W Brooks, ${ }^{1} \mathrm{~A}$ Burgess, ${ }^{3} \mathrm{~J}$ Wilson, ${ }^{1} \mathrm{~J}$ D Fortenberry. ${ }^{1}$ Indiana University School of Medicine, Indianapolis, USA; ${ }^{2}$ The Ohio State University, USA; ${ }^{3}$ UUPUI, USA

Background Little is known about how and where an adolescent lives and spends time relates to her health-related behaviours. Social disorder, or where crime occurs, is associated with various health outcomes but few have considered its association with adolescent

\section{SCHOOL DAY}

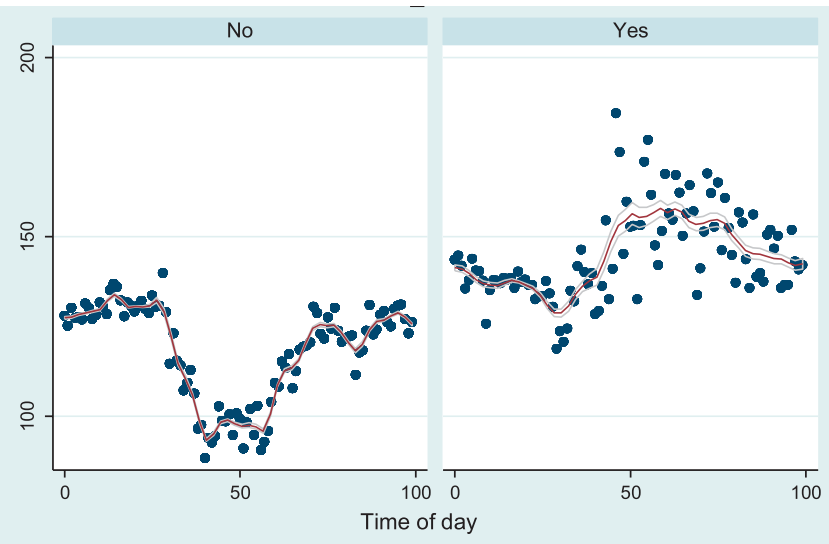

Abstract 01-S11.05 Figure 1 Average $200 \mathrm{~m}$ crime counts by time of day/day of week and self-reported sexual intercourse in the last 30 days. 